\title{
Equatorial orbits and imaging of hairy cubic Galileon black holes
}

\author{
K. Van Aelst๑* \\ Max Planck Institute for Gravitational Physics (Albert Einstein Institute), \\ Am Mühlenberg 1, 14476 Potsdam, Germany \\ and Laboratoire Univers et Théories, Observatoire de Paris, Université PSL, CNRS, Université de Paris, \\ 5 place Jules Janssen, 92190 Meudon, France \\ E. Gourgoulhon $\circledast^{\dagger}$ \\ Laboratoire Univers et Théories, Observatoire de Paris, Université PSL, CNRS, Université de Paris, \\ 5 place Jules Janssen, 92190 Meudon, France \\ F. H. Vincent ${ }^{*}$ \\ LESIA, Observatoire de Paris, Université PSL, CNRS, Sorbonne Université, Université de Paris, \\ 5 place Jules Janssen, 92190 Meudon, France
}

(Received 24 June 2021; accepted 1 November 2021; published 13 December 2021)

\begin{abstract}
Null and timelike equatorial orbits are investigated in a family of hairy black holes in the cubic Galileon theory. These include rotating generalizations of static black hole metrics supporting a time-dependent scalar field. Depending on the coupling and rotation, the properties of the geodesics expectedly deviate from general relativity. In particular, it is found that stable circular geodesics only exist below a critical coupling, which is related to the existence of an outermost stable circular orbit. Focusing on the strong-field region, images of an accretion disk are also produced to highlight tendencies that would constrain the model given further accurate observations of supermassive black holes.
\end{abstract}

DOI: 10.1103/PhysRevD.104.124034

\section{INTRODUCTION}

Trajectories of stars and images of accretion disks orbiting black holes provide some of the main observables to test strong-field gravity $[1,2]$. Such observational data are collected by complex instruments like the interferometer GRAVITY [3] and the Event Horizon Telescope (EHT) [4], which mainly focus on the supermassive black holes Sgr A* and M87* [5-8]. The theoretical predictions for these observables have been worked out within more or less exotic frameworks: Kerr black holes (e.g., [9]), rotating black holes dressed with a complex scalar hair [10,11], boson stars [9,12-14], alternative black holes [15-24], wormholes $[9,25]$, naked singularities [26], and binary systems $[27,28]$. Eventually, such analyses help constrain

\footnotetext{
*karim.vanaelst@aei.mpg.de

†eric.gourgoulhon@obspm.fr

frederic.vincent@obspm.fr
}

Published by the American Physical Society under the terms of the Creative Commons Attribution 4.0 International license. Further distribution of this work must maintain attribution to the author(s) and the published article's title, journal citation, and DOI. Open access publication funded by the Max Planck Society. the nature of the observed objects [2], but also the theory of gravity within which they are modeled [29-31].

It must be noted though that unequivocally excluding a given model is a complex endeavor in most cases. Current instrumental limitations and extremely large parameter spaces (describing e.g., the emission flow or sources of noise) require one to rely on simplifying prescriptions [32] to realize and interpret observations, such as the EHT reconstructed image. This leaves too much uncertainty to draw definitive conclusions today, and extensive studies are needed to explore significant parts of the parameter spaces and guess which observations could be decisive in discriminating some given models. But the understanding of astrophysical black holes gradually progresses by improving current instruments and analysis tools, and developing ideas for future enhanced observations.

In this context, the present paper focuses on the characteristic features and preliminary constraints arising from the strong-field observables of a family of black hole spacetimes within the cubic Galileon theory. The ("covariant generalized") Galileons are scalar-tensor theories which coincide with Horndeski theories in four dimensions, meaning that they are the most general scalar-tensor theories leading to second-order field equations [33-38]. Galileons thus provide a relevant framework to search for observable deviations from general relativity (GR), as most 
signatures of alternative theories are expected to be well described by scalar-tensor theories at least in some effective range. In particular, the cubic Galileon emerges from effective formulations of higher-dimensional theories, either in the decoupling limit of braneworld models such as the popular five-dimensional Dvali-Gabadadze-Porrati (DGP) model [34,39-42], or from Kaluza-Klein compactification of Lovelock theory $[43,44]$. Explicitly, its vacuum action writes

$S[g, \phi]=\int\left[\zeta(R-2 \Lambda)-\eta(\partial \phi)^{2}+\gamma(\partial \phi)^{2} \square \phi\right] \sqrt{|\operatorname{det} g|} d^{4} x$,

where $(\partial \phi)^{2} \equiv \nabla_{\mu} \phi \nabla^{\mu} \phi$ and $\zeta, \eta$, and $\gamma$ are coupling constants.

It is the simplest of Galileons with higher-order derivatives, and it is compatible with the observed speed of gravitational waves [45-48]. As a well-motivated, consistent theory, the cubic Galileon has been investigated in various contexts, from laboratory tests [49] to cosmology [50-54]. Besides current observations of supermassive black holes, further interest in the characteristics of cubic Galileon black holes comes from the fact that, together with most shift-symmetric ${ }^{1}$ Horndeski theories, the cubic Galileon is subject to a "no-scalar-hair" theorem: In the asymptotically flat framework, the only static, spherically symmetric black hole metric and scalar field are the Schwarzschild metric together with a trivial scalar field [55-58] (see also [59] for an extension to slow rotation and [60] for stars). Consequently, modified gravity effects can only occur in systems breaking one of these hypotheses. Indeed, one does obtain non-GR metrics coupled to nontrivial scalar hair when enforcing all hypotheses except the stationarity of the scalar field. Such a minimal violation of the hypotheses is possible when the scalar field features a linear time dependence [61]

$$
\phi=q t+\Psi
$$

where $q$ is a nonzero constant and $\Psi$ is time independent.

The shift symmetry is what makes a linear time dependence compatible with a static and spherically symmetric metric. Such a configuration was considered in various contexts such as cosmology, and the linear time dependence was physically interpreted as a first-order approximation to a slowly evolving scalar field $[53,54,62,63]$. Besides, using ansatz (2) (with $\Psi$ depending not only on the radial but also the angular coordinate), previous numerical work [64] produced rotating generalizations of hairy static and spherically symmetric solutions derived in the cubic Galileon theory [65]. At the level of the metric, these

\footnotetext{
${ }^{1}$ Shifting the scalar field by a constant $(\phi \rightarrow \phi+$ constant $)$ preserves action (1).
}

rotating black holes significantly deviate from Kerr spacetime, implying possibly observable modified gravity effects in black hole environments.

Such hairy configurations were constructed as circular spacetimes, meaning that they were assumed to admit a quasi-isotropic coordinate system with respect to which the line element writes

$d s^{2}=-N^{2} d t^{2}+A^{2}\left(d r^{2}+r^{2} d \theta^{2}\right)+B^{2} r^{2} \sin ^{2} \theta(d \varphi-\omega d t)^{2}$.

Circular spacetimes represent a large subclass of stationary and axisymmetric spacetimes, and their quasiisotropic coordinates are well suited to study geodesics. Yet the compatibility of a circular metric (3) with a linear time dependence (2) is exact only in the nonrotating case, while errors arise as rotation increases and could become significant at high rotation. This is why these spacetimes are only considered at low and moderate rotation such that the errors on the solutions are negligible [64]. Despite such a restriction, these configurations allow us to observe nonperturbative effects of rotation (examples of which are still not so abundant in modified gravity, although relevant for astrophysical black holes which are expected to rotate).

The metrics were constructed to be asymptotically flat, as it is a natural hypothesis of the no-scalar-hair theorem. Yet this is realized by taking $\eta=\Lambda=0$ in the cubic Galileon model (1), leaving the scalar field ruled by the nonstandard "DGP term" $(\partial \phi)^{2} \square \phi$. This induces a nonstandard asymptotic behavior of the metric which yields a vanishing Komar mass at infinity. This theoretical fact is unusual, but astrophysically, it does not forbid the hole to feature an effective mass on finite distances. More precisely, we will see that there do exist stable orbits, yet only up to an OSCO (outermost stable circular orbit). OSCOs are not so unusual even in GR in the presence of a positive cosmological constant, and they can emerge on short enough scales to be astrophysically relevant (for instance, using the Schwarzschild-de Sitter metric outside of a galaxy, the OSCO is of the order of the intergalactic distance [66]). In the cubic Galileon case, studying the geodesics of the asymptotically flat hairy black holes will show that the OSCO actually emerges on an even shorter scale when the remaining parameters of the model are chosen so as to generate non-negligible metric deviations in the strong-field region. The asymptotically flat model itself is then strongly constrained by requiring stability of all the orbits that would be dominantly ruled by a central supermassive black hole.

Note however that nonzero couplings $\eta$ and $\Lambda$ lead to Schwarzschild-de Sitter asymptotics in the static case [65], which generically restore a large OSCO compatible with observations. Despite different asymptotic properties, models with nonzero $\eta$ and $\Lambda$, and asymptotically flat solutions, might present analogous dependences on the DGP coupling 


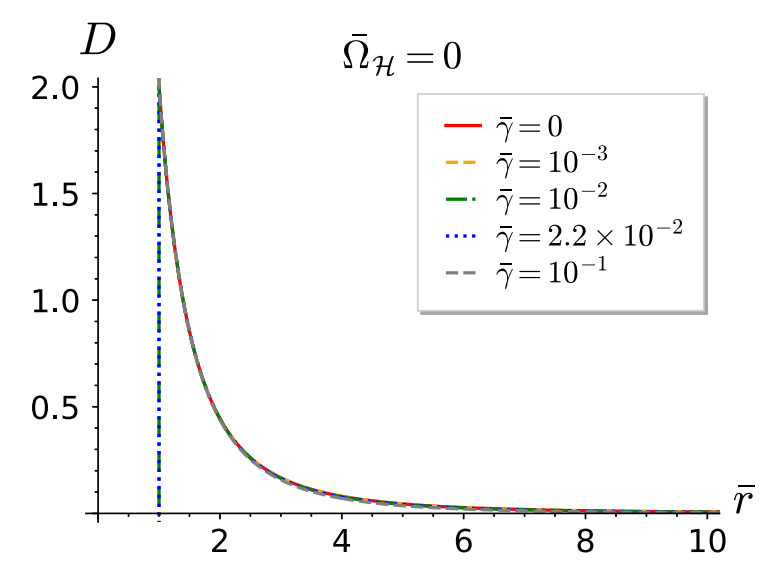

(a) Radial profile of function $D$ whose positivity allows (possibly superluminal) circular geodesics.

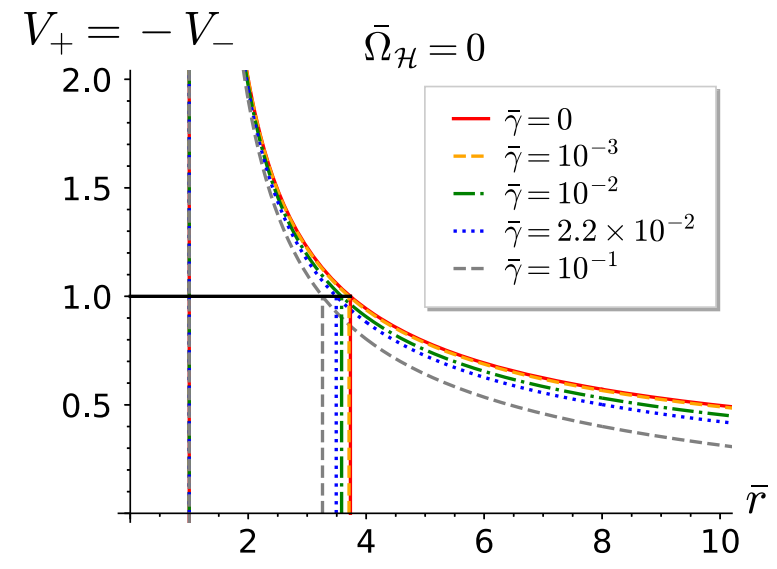

(b) Velocities and photon rings.

FIG. 1. Radial profiles of $D$ and the resulting velocities of circular geodesics in the static, spherically symmetric case (i.e., vanishing dimensionless angular velocity of the event horizon $\left.\bar{\Omega}_{\mathcal{H}}=r_{\mathcal{H}} \Omega_{\mathcal{H}}\right)$ for various couplings $(\bar{\gamma}=0$ corresponding to Schwarzschild spacetime). The lapse $N$ and its derivative are positive everywhere [see Fig. 2(a) of [64] ], so that $D>0$ implies that the denominator in (A11) is positive. Therefore, $V_{+}>0$ (prograde orbits) and $V_{-}=-V_{+}<0$ (retrograde orbits).

and on rotation, and share common observable characteristics in the strong-field region. This is why images of an accretion disk orbiting the asymptotically flat black holes are also presented below, while future work will construct asymptotically de Sitter solutions to assess such similarities (and more generally to identify degeneracies of the observables within the cubic Galileon model).

If some strong-field characteristics of the asymptotically flat configurations turned out particularly interesting without being preserved for any nonzero $\eta$ and $\Lambda$, one might deal with the unusual large distance properties of the asymptotically flat model by relying on convenient mechanisms or fields, such as a second, "screened" scalar field $\chi$. Screened scalar fields are commonly invoked in cosmology, in different realizations of massive gravity and hence in Galileon theories [41] to recover the successful predictions of GR on short scales, e.g., solar system scales, while providing new relevant cosmological phenomenology in regard to dark energy. In standard screening mechanisms (Chameleon [67,68], Symmetron [69,70], and Vainshtein [42,71-73]), the mass of $\chi$ or its coupling to matter effectively decrease in regions of high matter density. Such processes are realized through nonstandard kinetic terms and/or appropriate couplings to standard model matter, such as Dirac spinors, Higgs scalars, or other fundamental fields [69]. Although the idea has not been considered elsewhere, these mechanisms might be adapted to the present asymptotically flat case, e.g., by introducing analogous interactions that suitably couple $\chi$ to the black hole hair $\phi$ (rather than to standard matter): $\chi$ would be screened where $\phi$ adopts its strong-field profile, i.e., the immediate vicinity of the black hole, while it would modify the geometry on larger distances, and in particular the location of the OSCO. Thus, the asymptotically flat case may provide an effective description of the strong-field region of more complete models that feature more standard weak-field properties thanks to a standard kinetic term for $\phi$, a cosmological constant $\Lambda$, or alternative fields or mechanisms.

The plan of the article is as follows. The properties of the equatorial timelike circular geodesics and photon rings (location, stability, and deviation from Kerr spacetime) are studied in the static, spherically symmetric case in Sec. II A, and the rotating case in Sec. II B. This leads us to compute the images of an accretion disk orbiting the static, spherically symmetric black holes in Sec. III C, and the rotating black holes in Sec. III C. Notations and general results on equatorial geodesics in quasi-isotropic coordinates are summarized in the Appendix.

\section{ORBITS AROUND CUBIC GALILEON BLACK HOLES}

\section{A. Static and spherically symmetric case}

To study the geodesics of the cubic Galileon static and spherically symmetric black holes obtained in $[64,65]$, the procedure is to first characterize the circular geodesics. As detailed in the Appendix, regions of positive discriminant $D$ (A10) are first checked in Fig. 1(a) for different values of $\bar{\gamma}=q^{3} r_{\mathcal{H}} \gamma / \zeta$, where $r_{\mathcal{H}}$ is the radial coordinate of the event horizon. ${ }^{2}$ Function $D$ appears positive everywhere

\footnotetext{
${ }^{2}$ Recall that $\bar{\gamma}$ is the only remaining dimensionless coupling parametrizing deviations because $\eta$ and $\Lambda$ are set to 0 ; the values of $\bar{\gamma}$ considered in Fig. 1(a) are picked in the range leading to nonnegligible metric deviations from GR [64].
} 


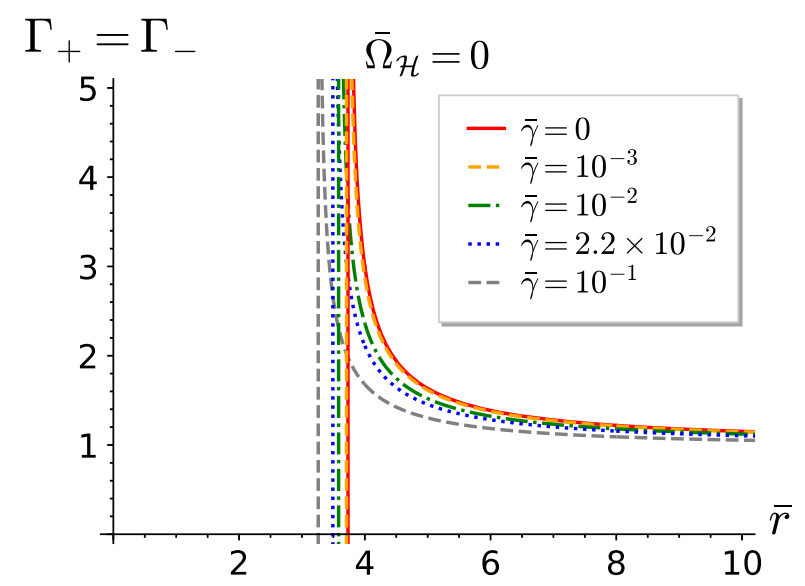

(a) Lorentz factor.

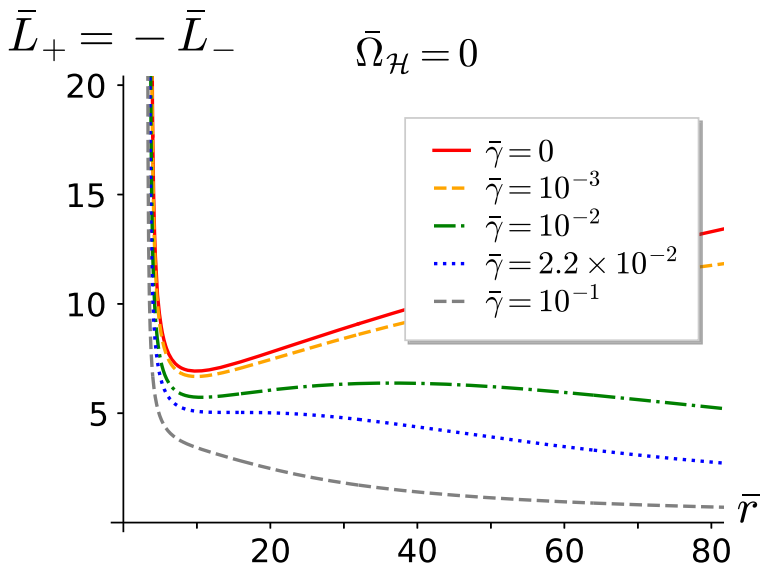

(b) Killing angular momentum.

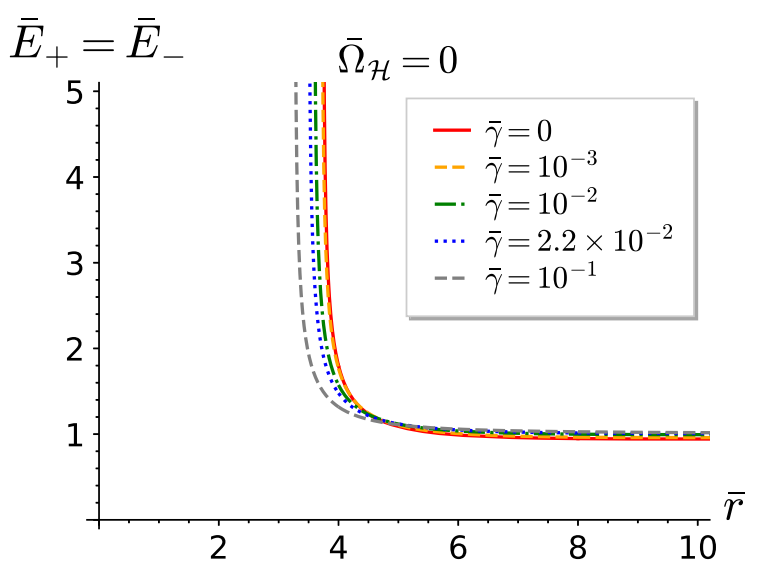

(c) Killing energy.

FIG. 2. Radial profiles of kinematic quantities measured by the zero angular momentum observer (ZAMO) for the timelike circular geodesics in the static, spherically symmetric case for various couplings. They all diverge at the photon ring (yet asymptotes are only plotted for the Lorentz factor).

down to the horizon, where it diverges because of division by the lapse $N$ which cancels at the horizon. Therefore, circular geodesics a priori exist everywhere for all couplings, but they necessarily become superluminal near the horizon according to (A11).

This is what Fig. 1(b) confirms: For each coupling $\bar{\gamma}$, the velocity diverges at the horizon so that there exists a photon ring (marked with a vertical line from 0 to 1 ), only beyond which timelike circular geodesics exist. Although $D$ does not vary much with the coupling in Fig. 1(a), the velocities more strongly depend on $\bar{\gamma}$ because function $B$ in the denominator of (A11) does vary [see Fig. 2(b) of [64], knowing that $B=A$ everywhere in spherical symmetry]. More precisely, at fixed radius, the velocity of the circular geodesic decreases with increasing coupling. As a consequence, the photon ring gets closer to the horizon as $\bar{\gamma}$ increases.

These results are related to the following facts mentioned in the Introduction. The metric functions $N$ and
$A=B$ converge faster to Minkowski at infinity as $\bar{\gamma}$ increases [64]. Therefore, at fixed radius away from the strong-field region, gravitation gets naively weaker as $\bar{\gamma}$ increases, so that the velocity of the circular geodesic must be smaller. In addition, for any $\bar{\gamma} \neq 0$, convergence to Minkowski spacetime is always much faster than that of Schwarzschild spacetime: $N$ and $A=B$ converge to 1 as $1 / r^{4}$ rather than $1 / r$, yielding a vanishing Komar mass at infinity [64]. As a result, velocities given by (A11) converge to zero like $r^{-\alpha / 2}$ with $\alpha=1$ in Schwarzschild spacetime and $\alpha=4$ in Galileon spacetimes.

Such asymptotic behaviors are highlighted in Fig. 2. In all cases, the Lorentz factor displayed in Fig. 2(a) logically converges to 1 . However, according to (A12), the Killing angular momentum per unit mass $\bar{L}$ displayed in Fig. 2(b) behaves like $r V \simeq r^{1-\alpha / 2}$; hence the divergence in Schwarzschild spacetime and convergence to zero for 


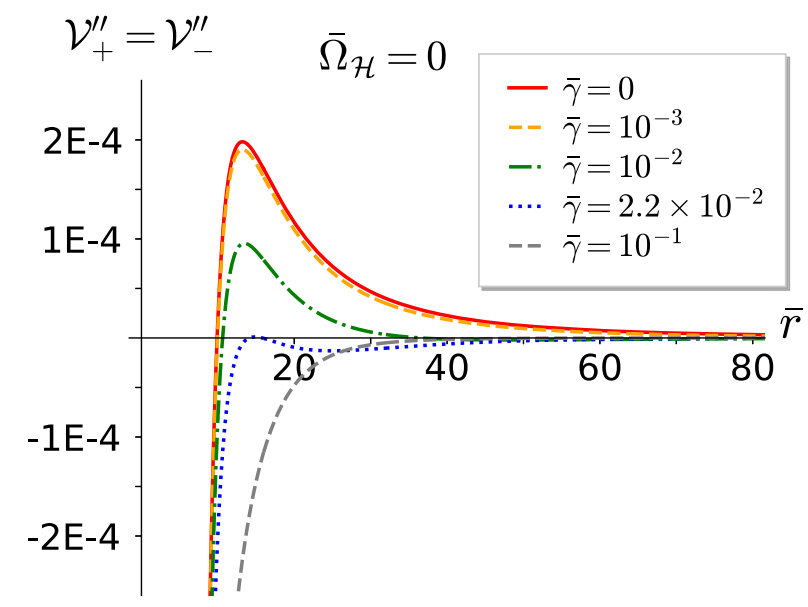

FIG. 3. Radial profile of $\mathcal{V}_{ \pm}^{\prime \prime}$; positivity determines the stability of the geodesics.

any $\bar{\gamma} \neq 0 .{ }^{3}$ Finally, $\bar{E}=\Gamma N$ converges to 1 in all cases in Fig. 2(c), which will hold true in the rotating case since function $\omega$ will converge to 0 like $1 / r^{3}$ regardless of whether $\bar{\gamma}$ is zero or not. At the photon ring, all the kinematic quantities displayed in Fig. 2 naturally diverge.

Figure 3 assesses the stability of circular orbits for various couplings based on the functions $\mathcal{V}_{ \pm}^{\prime \prime}$ given by (A14). As explained in the Appendix, their sign at a given radial coordinate $r_{0}$ is the same as $\mathcal{V}^{\prime \prime}\left(r_{0}, 1, \bar{E}_{ \pm}\left(r_{0}\right), \bar{L}_{ \pm}\left(r_{0}\right)\right)$ respectively. It appears that for any nonzero $\bar{\gamma}$, both an innermost stable circular orbit (ISCO) and OSCO exist. They respectively correspond to the smallest and greatest $r_{0}$ such that $\mathcal{V}_{ \pm}^{\prime \prime}\left(r_{0}\right)=0$. Since $\mathcal{V}_{ \pm}^{\prime \prime}$ globally decreases as $\bar{\gamma}$ increases, the ISCO radius increases while the OSCO decreases from infinity (where it is formally located in the Schwarzschild case $\bar{\gamma}=0$ ). In particular, the photon ring is always unstable because its location decreases when $\bar{\gamma}$ increases [Fig. 1(b)], so that it remains below the ISCO as in Schwarzschild spacetime.

The ISCO and OSCO eventually merge for a critical coupling $\bar{\gamma}^{c} \simeq 2.2 \times 10^{-2}$, beyond which no stable circular orbit exists anywhere. Therefore, the mere existence of stars orbiting black holes such as Sgr A* in a seemingly stable way requires $\bar{\gamma} \ll \bar{\gamma}^{c}$ for the present static Galileon black hole to be viable. The existence of a particularly close OSCO further constrains the model since e.g., the wellknown star $\mathrm{S} 2$ lies beyond $2500 M$, where $M \simeq 4 \times 10^{6} M_{\odot}$ is the observed mass of $\mathrm{Sgr} \mathrm{A}^{*}$. Although its orbit is noncircular, it indicates that a stable circular orbit exists between its apsides, and hence the OSCO must lie beyond. For instance, this further requires $\bar{\gamma} \ll 10^{-3}$.

\footnotetext{
${ }^{3}$ The numerical solutions contain information at infinity confirming this fact even for small couplings like $\bar{\gamma}=10^{-3}$ whose convergence to zero becomes apparent very far from the horizon.
}

Note though that a perturbation $\delta$ away from a circular orbit at some radius $r_{0}>r_{\mathrm{OSCO}}$ obeys equation

$$
\ddot{\delta}+\mathcal{V}^{\prime \prime}\left(r_{0}, 1, \bar{E}_{ \pm}\left(r_{0}\right), \bar{L}_{ \pm}\left(r_{0}\right)\right) \delta=0,
$$

so that the instability timescale is

$$
\tau\left(r_{0}\right)=1 / \sqrt{-\mathcal{V}^{\prime \prime}\left(r_{0}, 1, \bar{E}_{ \pm}\left(r_{0}\right), \bar{L}_{ \pm}\left(r_{0}\right)\right)} .
$$

Since $\mathcal{V}^{\prime \prime}$ asymptotically goes to zero as $r^{-6}, \tau$ diverges and rapidly becomes larger than the age of the Universe (e.g., around $30 r_{\mathrm{OSCO}}$ for $r_{\mathcal{H}}$ of the order of the Schwarzschild radius of $\mathrm{Sgr} \mathrm{A}^{*}$ and for $\bar{\gamma} \sim 10^{-2}$ ). Although this does not render the model viable, ${ }^{4}$ such an instability is weaker than in the standard cases featuring an OSCO (in Schwarzschild-de Sitter spacetime, $\tau$ converges to a finite value fixed by the cosmological constant). This goes along with the fact that this close OSCO is a singular artifact of the marginal combination $\eta=\Lambda=0$, and corroborates the idea that a much larger stability region would be restored with any additional Lagrangian terms or mechanism mentioned in the Introduction. With this in mind, the next section focuses on the effects of rotation on the orbits.

\section{B. Rotating case}

Rotation breaks spherical symmetry so that the "+" and "-" quantities are no longer equal or opposite, as shown in Fig. 4 (in which solid lines correspond to the analog quantities in Kerr spacetime). Yet all these quantities have the same behavior at the boundaries as in the static, spherically symmetric case.

Figure 4(a) shows that $V_{+}>0$ and $V_{-}<0$ still hold everywhere. However $V_{+}=-V_{-}$no longer does, so that there exist a prograde photon ring and a distinct retrograde one for each angular velocity $\bar{\Omega}_{\mathcal{H}}=r_{\mathcal{H}} \Omega_{\mathcal{H}}$. As in Kerr spacetime, prograde and retrograde velocities decrease as $\bar{\Omega}_{\mathcal{H}}$ increases, so that the prograde (resp. retrograde) ring radius decreases (resp. increases). The dependence on $\bar{\Omega}_{\mathcal{H}}$ yet seems stronger in Kerr spacetime, meaning e.g., that the prograde ring radius decreases faster than for any nonzero $\bar{\gamma}$. Since the photon ring of the static, spherically symmetric Galileon spacetime is below that of Schwarzschild spacetime, the relative positions of the Kerr and Galileon prograde rings are inverted for some $\bar{\Omega}_{\mathcal{H}}(\simeq 0.03$ for $\bar{\gamma}=10^{-2}$ ). On the contrary, the Kerr retrograde ring grows away from its Galileon counterpart.

The fact that the dependence on $\bar{\Omega}_{\mathcal{H}}$ is qualitatively the same in Kerr and Galileon spacetimes, but stronger in the former, also applies to $\bar{L}$ [Fig. 4(b)], $\bar{E}$ [Figs. 4(c) and 4(d)],

\footnotetext{
${ }^{4}$ This would not allow us to observe today distant bounded eccentric orbits like S2, but only quasicircular orbits very slowly drifting away which would have never experienced any strong radial perturbation.
} 


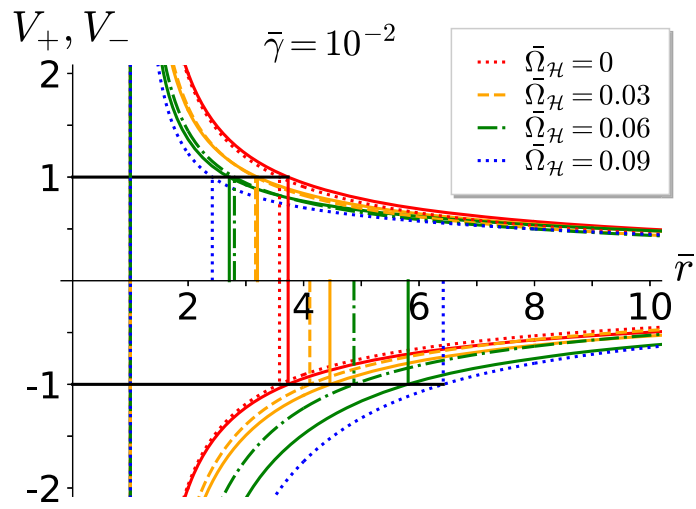

(a) Velocities $\left(V_{+}>0, V_{-}<0\right)$ and photon rings.

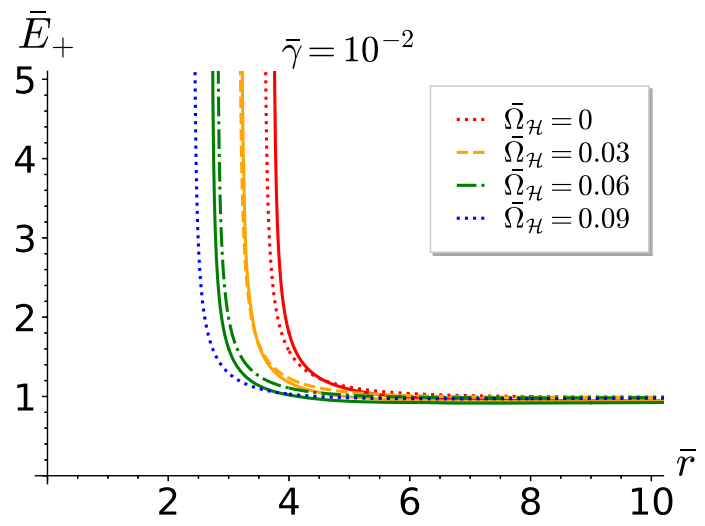

(c) Energy of prograde geodesics.

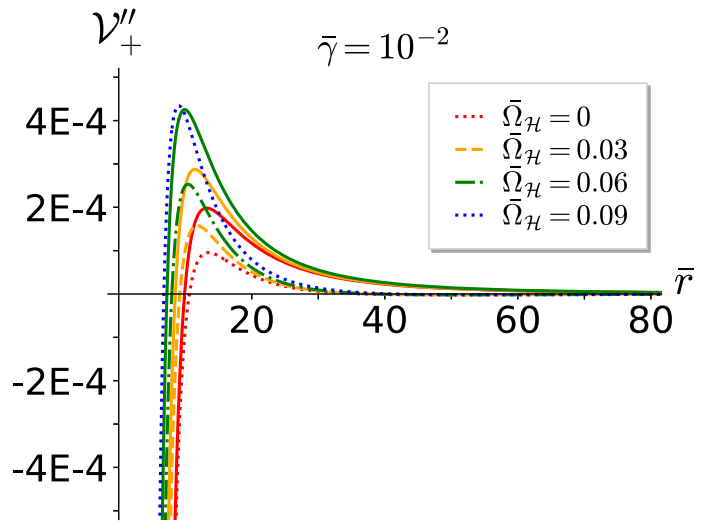

(e) Stability of prograde orbits from function $\mathcal{V}_{+}^{\prime \prime}$.

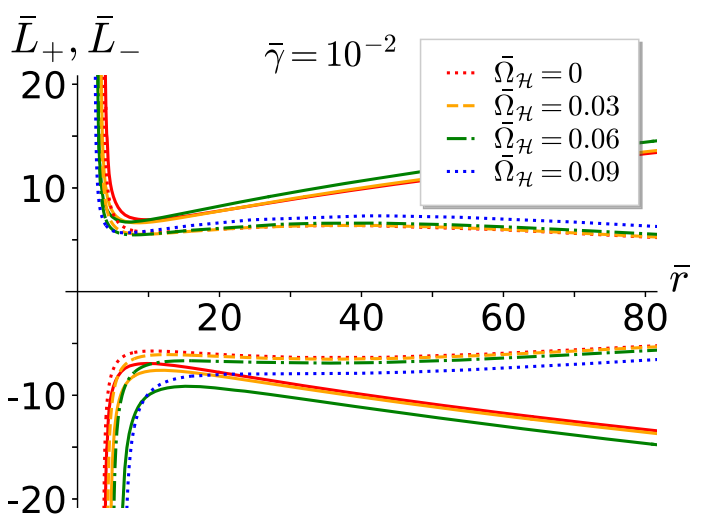

(b) Angular momentum $\left(\bar{L}_{+}>0, \bar{L}_{-}<0\right)$.

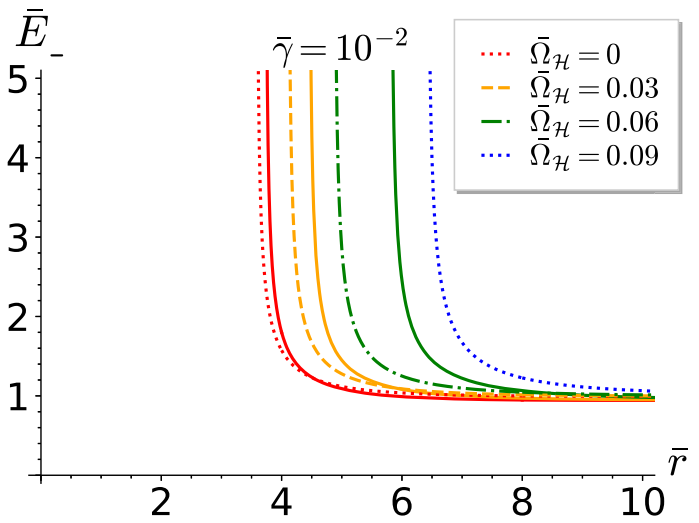

(d) Energy of retrograde geodesics.

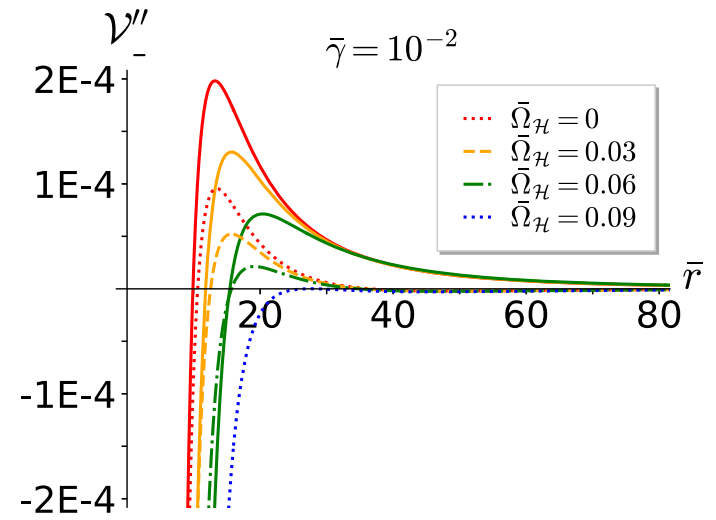

(f) Stability of retrograde orbits from function $\mathcal{V}_{-}^{\prime \prime}$.

FIG. 4. Kinematic quantities measured by the ZAMO and stability of the timelike circular geodesics for different angular velocities $\bar{\Omega}_{\mathcal{H}}=r_{\mathcal{H}} \Omega_{\mathcal{H}}$ at fixed coupling $\bar{\gamma}=10^{-2}<\bar{\gamma}^{c}$. For comparison, the profile in Kerr spacetime is plotted as a solid line with the same color for any fixed $\bar{\Omega}_{\mathcal{H}}$.

and $\mathcal{V}_{ \pm}^{\prime \prime}$ [Figs. 4(e) and 4(f)]. Besides, $\mathcal{V}_{+}^{\prime \prime}$ (resp. $\left.\mathcal{V}_{-}^{\prime \prime}\right)$ globally increases (resp. decreases) as $\bar{\Omega}_{\mathcal{H}}$ increases. Therefore, both the prograde ISCO and retrograde OSCO (resp. prograde OSCO and retrograde ISCO) radii decrease (resp. increase) with rotation. Interestingly, since the ISCO radius of static, spherically symmetric Galileon black holes is beyond Schwarzschild's ISCO, and Kerr's retrograde ISCO increases faster with $\bar{\Omega}_{\mathcal{H}}$, the relative positions of the Kerr and Galileon retrograde ISCOs are inverted for some $\bar{\Omega}_{\mathcal{H}}\left(\simeq 0.06\right.$ for $\left.\bar{\gamma}=10^{-2}\right)$. 


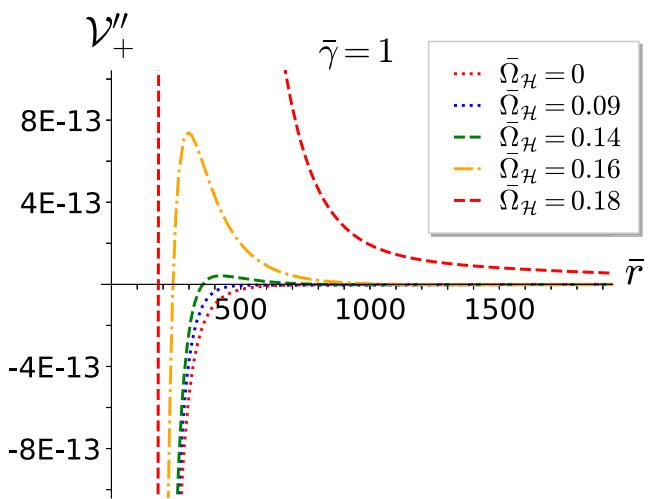

(a) Stability of prograde orbits for $\bar{\gamma}=1>\bar{\gamma}^{c}$.

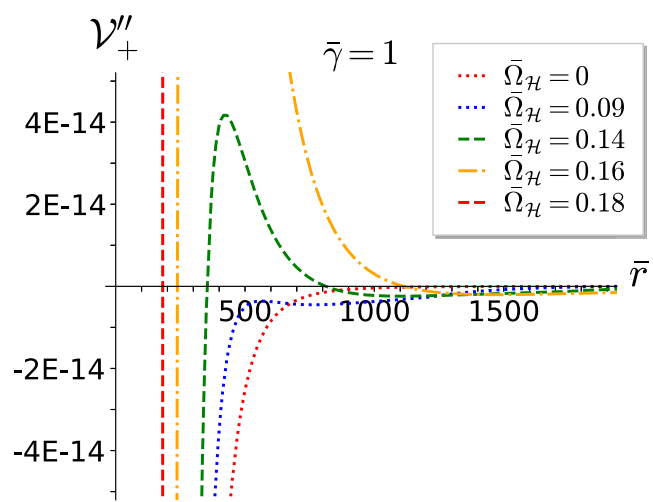

(b) Enlargement of Fig. 5(a).

FIG. 5. Rotation restores prograde stable orbits.

Furthermore, sufficiently high $\bar{\Omega}_{\mathcal{H}}$ makes it possible for $\mathcal{V}_{+}^{\prime \prime}$ to become positive even for $\bar{\gamma}$ greater than the critical coupling $\bar{\gamma}^{c} \simeq 2.2 \times 10^{-2}$; this is illustrated in Fig. 5(a) for $\bar{\gamma}=1>\bar{\gamma}^{c}$. Therefore, for each coupling $\bar{\gamma}>\bar{\gamma}^{c}$, there is a minimal angular velocity beyond which stable orbits reappear, yet only prograde ones. On the contrary, as $\bar{\Omega}_{\mathcal{H}}$ increases, the Galileon retrograde ISCO and OSCO eventually merge for a critical angular velocity $\bar{\Omega}_{\mathcal{H}}^{c}(\simeq 0.09$ for $\bar{\gamma}=10^{-2}$ ), beyond which no stable retrograde orbit exists anywhere. Therefore, the fact that stars stably orbit Sgr A* in both directions ${ }^{5}$ leads to even tighter constraints than $\bar{\gamma} \ll \bar{\gamma}^{c}$ if Sgr $\mathrm{A}^{*}$ is modeled as a rotating black hole.

\section{IMAGES OF CUBIC GALILEON BLACK HOLES}

\section{A. Principle of ray tracing}

In the present section, images of an accretion disk orbiting the black holes are computed numerically. This is again motivated by the idea that the present model may capture the significant characteristics of more complete, better-behaved models. Computations are performed by the free, opensource ray-tracing code GYOTO [74], which features an efficient approach to integrate the geodesic equations from the knowledge of the $3+1$ quantities decomposing a numerical metric [75]. In our case, the shift $\beta$ and spatial metric ${ }^{3} g$ corresponding to the quasi-isotropic metric (3) are

$$
\begin{gathered}
\beta=-\omega \partial_{\phi}, \\
{ }^{3} g=\operatorname{diag}\left(A^{2}, A^{2} r^{2}, B^{2} r^{2} \sin ^{2} \theta\right) .
\end{gathered}
$$

Images are computed in the following way. An explicit model of accretion flow is set around the black hole. ${ }^{6}$

\footnotetext{
${ }^{5}$ The spin direction of $\operatorname{Sgr} \mathrm{A}^{*}$ is unknown.

${ }^{6}$ Rough estimates confirmed by simple exact models of accretion disks show that the gravitational influence of an accretion disk is usually completely negligible with respect to the black hole. Thus, the vacuum black hole metrics are still valid in the presence of an accretion disk. See Sec. 6.5 of [1] for quantitative arguments.
}

A telescope set in the numerical metric mimics the observing wavelength $(1.3 \mathrm{~mm})$, the distance $(16.9 \mathrm{Mpc})$, field of view (120 $\mu \mathrm{as})$, and orientation of the Event Horizon Telescope with respect to M87* (the black hole being set at the origin and the disk lying in the equatorial plane $\theta=90^{\circ}$, the colatitude of Earth is $\theta=160^{\circ}$, while the vertical axis of the screen of the EHT is rotated by $110^{\circ}$ clockwise from the projection on the screen of the spin axis of the disk). Each pixel of its focal screen corresponds to a spatial direction, which uniquely defines the initial tangent vector of a null affinely parametrized geodesic. The latter is integrated backward in time until a stopping condition is met; e.g., the photon gets too close to the event horizon, or definitely leaves the strong-field region. Otherwise, every time the geodesic crosses the accretion disk, the radiative transfer equations ruling the specific intensity are integrated along the segment lying within the disk. The cumulated specific intensity is eventually plotted on the initial pixel. Yet, determining the nature and properties of a compact object based on the image of its accretion flow is a very degenerate inverse problem $[19,26,76]$. This is for instance evidenced in Ref. [13] in which the same model of the accretion disk is set around a boson star and a black hole: The deviations between the resulting images are very subtle although the natures of the accreting objects are very different. Furthermore, the resolution of present and future instruments like the EHT is limited, making it even harder to distinguish subtle features. ${ }^{7}$

Then, the purpose of numerical images is not systematically to check whether the image constructed by the EHT [8] can be reproduced for different accreting compact objects. This indeed requires costly general relativistic magnetohydrodynamics (GRMHD) simulations, together with a model of the EHT itself. Instead, strong efforts are made to propose fairly simple and yet realistic models

\footnotetext{
${ }^{7}$ Regarding the particular problem of distinguishing boson stars from black holes, see [14] for possibly detectable deviations arising from dynamical effects revealed by $3 \mathrm{D}$ general relativistic magnetohydrodynamics simulations.
} 


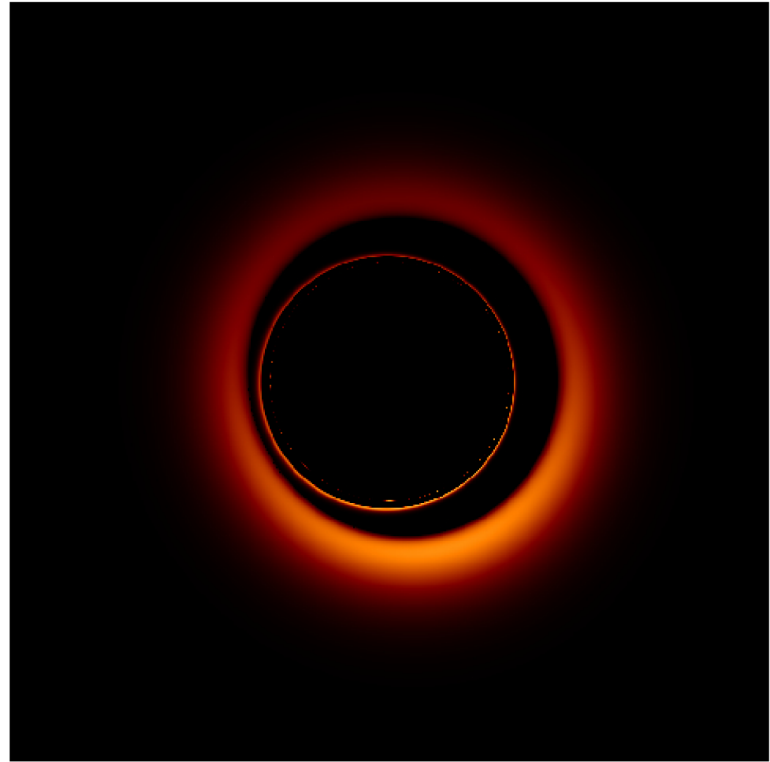

(a) $\left(\bar{\gamma}, \bar{\Omega}_{\mathcal{H}}\right)=(0,0)$ (Schwarzschild)

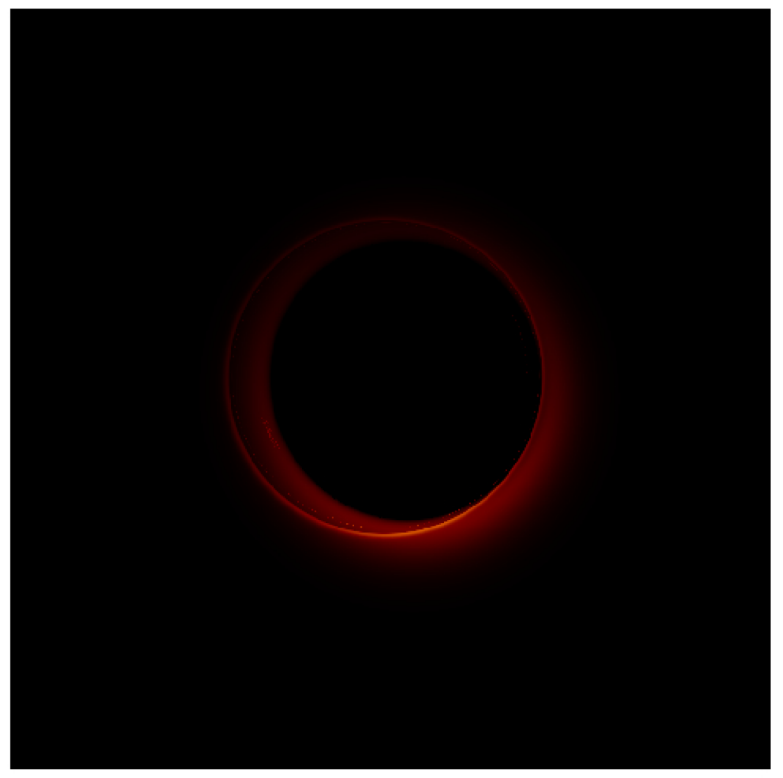

$6.98 \mathrm{e}-08$

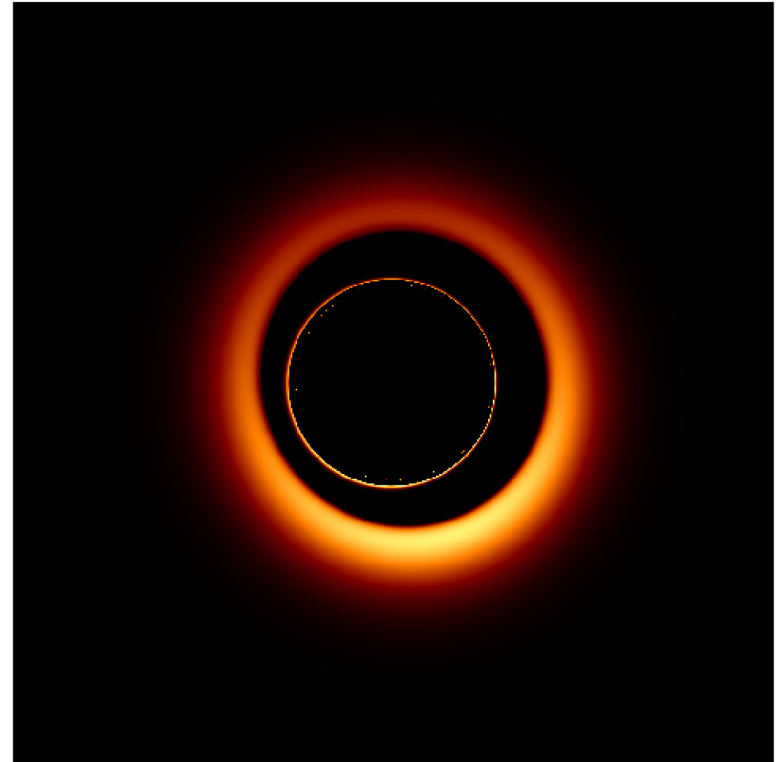

(b) $\left(\bar{\gamma}, \bar{\Omega}_{\mathcal{H}}\right)=\left(10^{-2}, 0\right)$ (static Galileon)
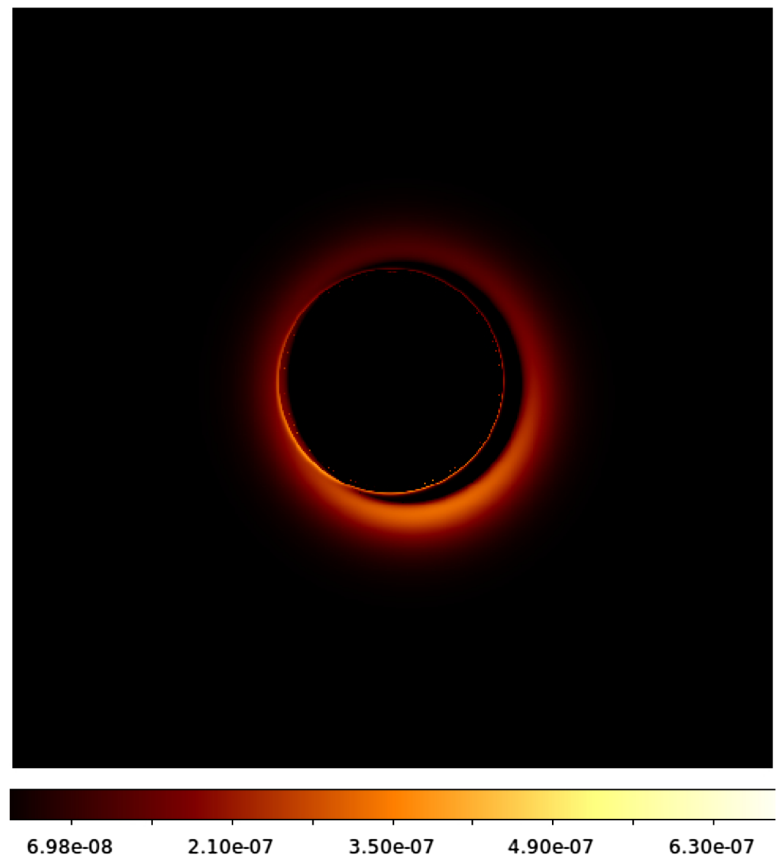

(d) $\left(\bar{\gamma}, \bar{\Omega}_{\mathcal{H}}\right)=\left(10^{-2}, 0.07\right)$ (rotating Galileon)

FIG. 6. Images at $1.3 \mathrm{~mm}$ produced by a thick accretion disk orbiting black holes for various couplings $\bar{\gamma}$ and angular velocity $\bar{\Omega}_{\mathcal{H}}$ (fixed horizon radius $r_{\mathcal{H}}=M_{M 87^{*}} / 2$ ). The field of view equals $120 \mu$ as. The linear scale of the specific intensity $I_{\nu}$ is provided in Systeme International $(\mathrm{SI})$ units $\left(I_{\nu}=4 \times 10^{7} \mathrm{SI}\right.$ corresponds to a brightness temperature $\left.\sim 25 \times 10^{9} \mathrm{~K}\right)$.

of accretion disks [9,77-79]. In particular, such models are assumed to be good approximations of stable steady solutions of the GRMHD equations. Comparing the resulting images for different compact objects provides a more efficient and still relevant method to evaluate how degenerate the problem is. The hope is that such an approach should help isolate the causes of differences between images, e.g., being able to guess the nature and amplitudes of the modifications that result from changing the accretion model or the theory used to describe the whole system.

As a result, a simple model of the accretion disk, recently introduced in [9], is used in the sections below. Like Sgr A*, supermassive black hole M87* features a very lowluminosity accretion flow, revealing an inefficient radiative cooling and hence a high temperature. It is consistently 
modeled as a low accretion rate, geometrically thick, optically thin $\operatorname{disk}^{8}[80]$. Besides these properties, only the thermal synchrotron emission is computed, following a method exposed in [81]. In the end, the complete model is described by very few input parameters: the opening angle and inner edge of the disk (which is set at the ISCO in our case), the magnetization parameter (which determines the ambient magnetic field strength), and the electron density and temperature at the inner edge (which determine the density and temperature profiles).

\section{B. Static and spherically symmetric case}

To connect with Sec. II, the black holes in Fig. 6 all have the same radius, namely the Schwarzschild radius of M87* (in quasi-isotropic coordinates, i.e., $r_{\mathcal{H}}=M_{M 87^{*}} / 2$ where $M_{M 87^{*}} \simeq 6.5 \times 10^{9} M_{\odot}$ ). It is actually more natural to manipulate the horizon radius than any notion of mass: Because of the non-Schwarzschild asymptotics of the Galileon black holes recalled in Sec. II A, there is no relevant mass parameter that can be extracted from star trajectories (through Kepler's law) or surface integrals (such as the Komar or Arnowitt-Deser-Misner masses) in the weak-field region, since its value could not be compared in any meaningful way to that of a Schwarzschild black hole (recall for instance that these Galileon black holes feature a vanishing Komar mass at spatial infinity, and yet admit stable orbits). It is thus equally legitimate to make comparisons based on parameters specific to the strongfield region such as the horizon, light ring and ISCO radii, and the characteristics of the images described below.

In the nonrotating case, Figs. 6(a) and 6(b) compare the Schwarzschild limit $\bar{\gamma}=0$ to a static and spherically symmetric Galileon black hole below the critical coupling. In both cases, the ISCO radius is read from Fig. 3. The asymmetries within each image come from the configuration of the EHT with respect to the accretion disk. More precisely, the $110^{\circ}$ clockwise rotation of the vertical axis of the screen from the projected spin axis of the disk explains the position on the images of the brighter spot that results from the relativistic beaming and blueshift affecting the part of the disk rotating toward the screen. Besides, the inside luminous ring corresponds to the secondary- and higher-order images, which asymptotically accumulate in the direction of the light ring. Rather than being centered within the primary image of the disk, this ring appears shifted toward the top of the spin axis because of the inclination angle $\theta=160^{\circ}$ of the EHT observer (the disk is almost seen from below).

This ring gets smaller with respect to the primary image as $\bar{\gamma}$ increases because the ISCO radius increases (Fig. 3) while the light ring decreases [Fig. 1(b)]. Explicitly, in the

\footnotetext{
${ }^{8}$ An accretion disk is geometrically (resp. optically) thin when the opening angle (resp. optical depth) is smaller than 1. It is geometrically (resp. optically) thick otherwise.
}

Schwarzschild case, the internal diameter of the primary image is $D \simeq 50 \mu$ as while the diameter of the secondary ring is $d \simeq 42 \mu$ as, which is compatible with the EHT image [8]. In the Galileon case, $D \simeq 46 \mu$ as while $d \simeq 34 \mu$ as. Besides, the image is globally brighter as $\bar{\gamma}$ increases. This is consistent with the fact that asymptotic convergence to Minkowski is faster as $\bar{\gamma}$ increases (as recalled in Sec. II A). In other terms, the strong-field region shrinks as $\bar{\gamma}$ increases, so that most light rays undergo a weaker gravitational redshift. Therefore, if one considered a Galileon black hole with e.g., a 1.12 times greater radius, so as to fit the internal and secondary diameters close enough to the Schwarzschild values (the deviation of their ratio being possibly undetectable by the resolution of the $\mathrm{EHT}^{9}$ ), the corresponding image would get even brighter than Fig. 6(b). One could accordingly reduce the density parameter to avoid tension with the observed luminosity. Yet the fact would remain that the ISCO radius would be even greater than it already is with respect to its Schwarzschild analog when the horizon radii are the same [Fig. 3]. It is hardly conceivable that the ISCO radius of M87* could be estimated by other means than EHT-type observations, so that no incompatibility related to this parameter could be exhibited. On the other hand, if images of Sgr A* were obtained, tensions about the ISCO location should arise based on the high precision astrometric observations made by the instrument GRAVITY which detected "flares" [6] close to Schwarzschild ISCO, knowing that such bright spots are expected to materialize near the inner edge of the accretion disk of Sgr A*.

\section{Rotating case}

Figures 6(c) and 6(d) compare a Kerr black hole to a rotating Galileon black hole sharing the same angular velocity. In both cases, the inner edge of the disk is set at the prograde ISCO, which is read from Fig. 4(e). Besides, the inside ring intersects the primary image, meaning that the ISCO is close enough to the light ring to allow photons to cross the disk at least a second time. This is consistent with the fact that the prograde ISCO decreases [Fig. 4(e)] faster than the prograde light ring [Fig. 4(a)] as $\bar{\Omega}_{\mathcal{H}}$ increases. As in the static case, the image is globally brighter as $\bar{\gamma}$ increases. But at a fixed $\bar{\gamma}$, the images get darker as $\bar{\Omega}_{\mathcal{H}}$ increases because the strong-field region expectedly expands with rotation.

As illustrated by Fig. 7, such tendencies leave room for a black hole with moderately low rotation, and radius greater

\footnotetext{
${ }^{9}$ Actually, in order to reproduce even more closely the diameters, and hence their ratio, one could fine-tune the dimension of the internal diameter of the primary image by considering a more realistic scenario involving accreting matter on noncircular trajectories below the ISCO. In this sense, the secondary ring is more reliable as an observable of the gravitational field as it weakly depends on the boundaries and physical properties of the accretion process.
} 


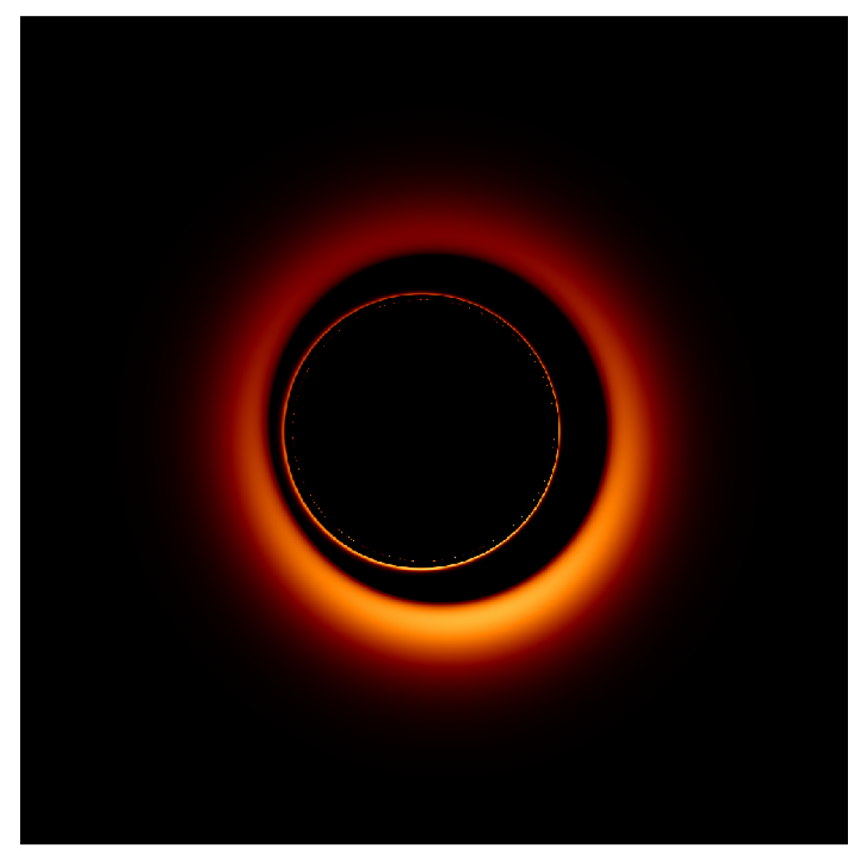

$6.98 \mathrm{e}-08 \quad 1.40 \mathrm{e}-07 \quad 2.10 \mathrm{e}-07 \quad 2.80 \mathrm{e}-07 \quad 3.50 \mathrm{e}-07 \quad 4.20 \mathrm{e}^{-07} \quad 4.90 \mathrm{e}-07 \quad 5.60 \mathrm{e}-07 \quad 6.30 \mathrm{e}-07$

FIG. 7. Case $\left(\bar{\gamma}, \bar{\Omega}_{\mathcal{H}}\right)=\left(10^{-2}, 0.03\right)$ such that $r_{\mathcal{H}}=1.2 M_{M 87^{*}} / 2$. To compare with Fig. 6(a).

than $M_{M 87^{*}} / 2$, to closely mimic Schwarzschild Fig. 6(a) in terms of dimensions and brightness. Yet regarding the ISCO radius, the fit is expectedly not so good with respect to Schwarzschild (9\% and 50\% deviation on the prograde and retrograde ISCO respectively). Of course, clearing away degenerate tendencies and exhibiting significant incompatibilities would only be possible if images and more precise spin measurements of Sgr A* were available.

\section{CONCLUSION}

Investigating the geodesics of asymptotically flat cubic Galileon black holes exhibits nonviable characteristics when the coupling $\bar{\gamma}$ is in a range leading to non-negligible metric deviations from GR. As such, the model would be constrained to a negligible coupling $\bar{\gamma}$ for the OSCO to lie far enough to be compatible with distant stars observed around Sgr A*. Yet it is thought that constraints only based on the OSCO might be dismissed by restoring terms in Lagrangian (1), or invoking other appropriate fields, which would preserve the tendencies and strong-field characteristics of the asymptotically flat model. This is why images of accretion disks have also been computed, as these allow us to probe the close environment of a supermassive black hole. The dependence of this observable on coupling and rotation may lead to deviations from GR in terms of global brightness and relative dimensions of the luminous structures. Yet these may often be compensated by adjusting density and the horizon radius.
Proper constraints will hopefully arise from future images and precise measurements of the spin and ISCO location of $\operatorname{Sgr} \mathrm{A}^{*}$. The latter being one of the main targets of the EHT, knowledge about this system will keep increasing in the coming years. Besides probing the inner accretion flow [82,83], the observations from the EHT will contribute to evaluating its spin (while former estimations are based e.g., on quasiperiodic oscillations of the radio emission [84]). Indeed, EHT data were already used to establish bounds on the spin of M87* [85,86], and this will improve with further observations from the EHT [31]. Information on the spin may also be extracted from instruments of the Very Large Telescope such as the interferometer GRAVITY which can monitor faint stars very close to $\mathrm{Sgr} \mathrm{A}^{*}$ [87]. From a broader point of view, GRAVITY also contributes to a better understanding of the astrophysics of massive black holes [88], and such general knowledge will also improve in future decades thanks to the space-based gravitational-wave observatory LISA [89,90], which will extract precious and precise information from stellar-mass compact objects spiraling around massive black holes. On an even longer term, Ref. [91] proposes a space-based very long baseline interferometry experiment which would characterize the ring-shaped structures in images of black hole accretion flows with extremely high precision, measuring the diameter size to $0.04 \%$ accuracy, while the EHT is limited to approximately $10 \%$ accuracy. This highlights again the fact that constraining theories of gravity with massive black holes is a long-term enterprise, as determining the parameters of black holes and their accretion flow is a degenerate problem conditioned by instrumental accuracy and modeling limitations [92-94]. It is in particular delicate to predict when and which opportune combination of observations will provide inflexible constraints or could at least strongly disfavor a model.

The models considered in the present paper are not free of simplifications either, both in the metric and the model of the disk, which could be addressed in later work. As explained in the Introduction, the circular metric (3) is only exact in the static case. Reproducing the rotating solutions in a noncircular framework would allow us to reach accurate rapidly rotating solutions (possibly up to an extremal case). Such a general framework would also be used to construct the asymptotically de Sitter configurations corresponding to nonzero $\eta$ and $\Lambda$, and perform similar study and comparisons. Furthermore, in order to make precise quantitative comparisons between actual images and numerical predictions in a consistent way, more realistic models of a disk such as ion tori could be considered. The latter are also geometrically thick and optically thin structures, yet featuring more complex density and temperature profiles derived from first principles, as well as isotropic [77] or toroidal [79] magnetic fields, hence allowing us to explore other parts of the parameter space describing accretion disks. 


\section{ACKNOWLEDGMENTS}

K. V. and E. G. acknowledge support from the CNRS program 80PRIME-TNENGRAV.

\section{APPENDIX: EQUATORIAL GEODESICS IN QUASI-ISOTROPIC COORDINATES}

The present Appendix recalls useful results on particles freely moving in the equatorial plane of a circular spacetime described in terms of the quasi-isotropic coordinates (3) (see [95-97] and Secs. 4.6 and 4.7 of [98] for closely related discussions). Based on the unique parametrization

$$
\mathcal{C}: \lambda \mapsto\left(x^{\mu}(\lambda)\right)=(t(\lambda), r(\lambda), \pi / 2, \phi(\lambda))
$$

such that the 4-momentum of the particle is $p^{\mu}=\dot{x}^{\mu}$ (where a dot denotes differentiation with respect to the parameter $\lambda$ ), the geodesic equation

$$
\nabla_{p} p=0
$$

implies that

$$
\begin{gathered}
E=-p_{t} \text { is conserved along } \mathcal{C}, \\
L=p_{\phi} \text { is conserved along } \mathcal{C}, \\
\theta=\pi / 2 \text { is conserved along } \mathcal{C}, \\
\quad \frac{\dot{r}^{2}}{2}+\mathcal{V}(r, m, E, L)=0,
\end{gathered}
$$

where $m=\sqrt{-p^{2}}$ defines the mass of the particle, and the effective potential $\mathcal{V}$ is defined as

$\mathcal{V}(r, m, E, L)=\frac{1}{2 A^{2}}\left[m^{2}-\left(\frac{E-\omega L}{N}\right)^{2}+\left(\frac{L}{\mathrm{Br}}\right)^{2}\right]$.

Equation (A6) is merely an explicit version of the mass conservation equation ( $m$ is conserved along $\mathcal{C}$ ) taking advantage of the three other conservation equations (A3)-(A5). Thus, these four conservation equations are four necessary conditions for a curve $\mathcal{C}$ to describe an equatorial trajectory of a free particle. For noncircular orbits (i.e., $\dot{r} \neq 0$ almost everywhere), they are sufficient and have a unique solution provided that the initial sign of $\dot{r}$ is fixed and the initial coordinates $\left(t_{0}, r_{0}, \pi / 2, \phi_{0}\right)$, the Killing energy $E$, the Killing angular momentum $L$, and the mass $m$ satisfy $\mathcal{V}\left(r_{0}, m, E, L\right)<0$ and $E-\omega\left(r_{0}\right) L>0$ (to guarantee that the trajectory is initially causal, i.e., future oriented). For a circular geodesic at radial coordinate $r_{0}$, one has $\dot{r}=0$ so that $\mathcal{V}\left(r_{0}, m, E, L\right)=0$. Yet, as detailed in [97], the additional condition

$$
\mathcal{V}^{\prime}\left(r_{0}, m, E, L\right)=0
$$

is required besides Eqs. (A3)-(A5) to realize a geodesic instead of an arbitrary (possibly accelerated) circular orbit. Rather than $E$ and $L$, this constraint is usually formulated in terms of the spatial velocity $V$ measured by the $\mathrm{ZAMO}^{10}$ as

$$
\left(\frac{B^{\prime}}{B}+\frac{1}{r}\right) V^{2}-\frac{B r \omega^{\prime}}{N} V-\frac{N^{\prime}}{N}=0 .
$$

Roots exist if and only if the discriminant

$$
D=\left(\frac{B r \omega^{\prime}}{N}\right)^{2}+\frac{4 N^{\prime}}{N}\left(\frac{B^{\prime}}{B}+\frac{1}{r}\right)
$$

is non-negative, in which case one has

$$
V_{ \pm}(r)=\frac{\frac{B r \omega^{\prime}}{N} \pm \sqrt{D}}{2\left(\frac{B^{\prime}}{B}+\frac{1}{r}\right)} .
$$

A timelike circular geodesic (resp. photon ring) exists at $r$ when $V_{ \pm}$are defined at $r$ and at least one of them belongs to $(-1,1)$ (resp. $\{-1,1\}$ ) since the ZAMO necessarily measures a subluminal (resp. luminal) velocity. The corresponding Killing energy and angular momentum of the geodesic are

$$
\begin{gathered}
L_{ \pm}=\mathcal{E} B r V_{ \pm}, \\
E_{ \pm}=\mathcal{E}\left(N+B r \omega V_{ \pm}\right),
\end{gathered}
$$

where $\mathcal{E}$ is the energy measured by the ZAMO: For a massive particle, $\mathcal{E}=\Gamma m$ where $\Gamma$ is the Lorentz factor of the particle with respect to the ZAMO, while for a massless particle, $\mathcal{E}=h \nu$ where $\nu$ is the frequency measured by the ZAMO.

Finally, the radial equation (A6) expectedly provides stability criteria for circular geodesics based on convexity: For any perturbation to be bounded in some neighborhood of a geodesic at $r, \mathcal{V}^{\prime \prime}(r, m, E, L)$ must be positive. Since the values of $E$ and $L$ for a circular geodesic at $r$ are necessarily given by relations (A12) and (A13), one only has to study the sign of the two functions

$$
\mathcal{V}_{ \pm}^{\prime \prime}: r \mapsto \mathcal{V}^{\prime \prime}\left(r, m, E_{ \pm}(r), L_{ \pm}(r)\right)
$$

on the set on which the discriminant $D$ is non-negative. Actually, the expressions $\mathcal{V}_{ \pm}^{\prime \prime}(r)$ are homogeneous with

\footnotetext{
${ }^{10}$ The ZAMOs are characterized by a 4-velocity collinear to $\nabla t$.
} 
respect to $\mathcal{E}$, so that their sign does not depend on $\Gamma_{ \pm} m$ in the massive case or on $h \nu$ in the massless case. Therefore, the stability of the causal circular geodesics only depends on the sign of the two functions (A14) and corresponds to massive particles where $V_{ \pm}(r) \in(-1,1)$ and massless ones where $V_{ \pm}(r)= \pm 1$, regardless of whether the expressions used for $E_{ \pm}$and $L_{ \pm}$apply to a massive or a massless particle. These are therefore the two functions that are plotted in Sec. II to study the stability of circular geodesics in the cubic Galileon spacetimes.
[1] C. Bambi, Black Holes: A Laboratory for Testing Strong Gravity (Springer, New York, 2017).

[2] V. Cardoso and P. Pani, Testing the nature of dark compact objects: A status report, Living Rev. Relativity 22, 4 (2019).

[3] R. Abuter et al. (GRAVITY Collaboration), First light for GRAVITY: Phase referencing optical interferometry for the Very Large Telescope Interferometer, Astron. Astrophys. 602, A94 (2017).

[4] R. Abuter et al. (GRAVITY Collaboration), Imaging an Event Horizon: submm-VLBI of a Super Massive Black Hole, arXiv:0906.3899.

[5] R. Abuter et al. (GRAVITY Collaboration), Detection of the gravitational redshift in the orbit of the star S2 near the Galactic centre massive black hole, Astron. Astrophys. 615, L15 (2018).

[6] R. Abuter et al. (GRAVITY Collaboration), Detection of orbital motions near the last stable circular orbit of the massive black hole SgrA, Astron. Astrophys. 618, L10 (2018).

[7] S. Issaoun et al., The size, shape, and scattering of Sagittarius $\mathrm{A}^{*}$ at $86 \mathrm{GHz}$ : First VLBI with ALMA, Astrophys. J. 871, 30 (2019).

[8] K. Akiyama et al. (EHT-Collaboration), First M87 Event Horizon Telescope Results. I. The Shadow of the Supermassive Black Hole, Astrophys. J. Lett. 875, 1 (2019).

[9] F. H. Vincent, M. Wielgus, M. A. Abramowicz, E. Gourgoulhon, J. P. Lasota, T. Paumard, and G. Perrin, Geometric modeling of M87* as a Kerr black hole or a non-Kerr compact object, Astron. Astrophys. 646, A37 (2021).

[10] F. H. Vincent, E. Gourgoulhon, C. Herdeiro, and E. Radu, Astrophysical imaging of Kerr black holes with scalar hair, Phys. Rev. D 94, 084045 (2016).

[11] L. G. Collodel, D. D. Doneva, and S. S. Yazadjiev, Circular orbit structure and thin accretion disks around kerr black holes with scalar hair, Astrophys. J. 910, 52 (2021).

[12] M. Grould, Z. Meliani, F. H. Vincent, P. Grandclément, and E. Gourgoulhon, Comparing timelike geodesics around a Kerr black hole and a boson star, Classical Quant. Grav. 34, 215007 (2017).

[13] F. H. Vincent, Z. Meliani, P. Grandclément, E. Gourgoulhon, and O. Straub, Imaging a boson star at the Galactic center, Classical Quant. Grav. 33, 105015 (2016).

[14] H. Olivares, Z. Younsi, C. M. Fromm, M. De Laurentis, O. Porth, Y. Mizuno, H. Falcke, M. Kramer, and L. Rezzolla, How to tell an accreting boson star from a black hole, Mon. Not. R. Astron. Soc. 497, 521 (2020).
[15] T. Vetsov, G. Gyulchev, and S. Yazadjiev, Shadows of Black Holes in Vector-Tensor Galileons Modified Gravity, arXiv:1801.04592.

[16] M. Wang, S. Chen, and J. Jing, Shadow casted by a Konoplya-Zhidenko rotating non-Kerr black hole, J. Cosmol. Astropart. Phys. 10 (2017) 051.

[17] N. Tsukamoto, Black hole shadow in an asymptotically flat, stationary, and axisymmetric spacetime: The Kerr-Newman and rotating regular black holes, Phys. Rev. D 97, 064021 (2018).

[18] H.-M. Wang, Y.-M. Xu, and S.-W. Wei, Shadows of Kerrlike black holes in a modified gravity theory, J. Cosmol. Astropart. Phys. 03 (2019) 046.

[19] A. B. Abdikamalov, A. A. Abdujabbarov, D. Ayzenberg, D. Malafarina, C. Bambi, and B. Ahmedov, Black hole mimicker hiding in the shadow: Optical properties of the $\gamma$ metric, Phys. Rev. D 100, 024014 (2019).

[20] J. W. Moffat and V. T. Toth, Masses and shadows of the black holes Sagittarius A* and M87* in modified gravity, Phys. Rev. D 101, 024014 (2020).

[21] M. Khodadi, A. Allahyari, S. Vagnozzi, and D. F. Mota, Black holes with scalar hair in light of the Event Horizon Telescope, J. Cosmol. Astropart. Phys. 09 (2020) 026.

[22] A. Das, A. Saha, and S. Gangopadhyay, Shadow of charged black holes in Gauss-Bonnet gravity, Eur. Phys. J. C 80, 180 (2020).

[23] S.-W. Wei and Y.-X. Liu, Testing the nature of GaussBonnet gravity by four-dimensional rotating black hole shadow, Eur. Phys. J. Plus 136, 436 (2021).

[24] M. Guo and P.-C. Li, Innermost stable circular orbit and shadow of the 4D Einstein-Gauss-Bonnet black hole, Eur. Phys. J. C 80, 588 (2020).

[25] F. Lamy, E. Gourgoulhon, T. Paumard, and F. H. Vincent, Imaging a non-singular rotating black hole at the center of the Galaxy, Classical Quant. Grav. 35, 115009 (2018).

[26] R. Shaikh and P. S. Joshi, Can we distinguish black holes from naked singularities by the images of their accretion disks?, J. Cosmol. Astropart. Phys. 10 (2019) 064.

[27] P. V. P. Cunha, C. A. R. Herdeiro, and M. J. Rodriguez, Does the black hole shadow probe the event horizon geometry?, Phys. Rev. D 97, 084020 (2018).

[28] P. V. P. Cunha, C. A. R. Herdeiro, and M. J. Rodriguez, Shadows of exact binary black holes, Phys. Rev. D 98, 044053 (2018).

[29] D. Psaltis et al. (EHT Collaboration), Gravitational Test beyond the First Post-Newtonian Order with the Shadow of the M87 Black Hole, Phys. Rev. Lett. 125, 141104 (2020). 
[30] S. Völkel, E. Barausse, N. Franchini, and A. E. Broderick, EHT tests of the strong-field regime of general relativity, Classical Quant. Grav. 38, 21LT01 (2021).

[31] P. Kocherlakota et al. (EHT Collaboration), Constraints on black-hole charges with the 2017 EHT observations of M87*, Phys. Rev. D 103, 104047 (2021).

[32] A. E. Broderick et al., THEMIS: A parameter estimation framework for the Event Horizon Telescope, Astrophys. J. 897, 139 (2020).

[33] G. W. Horndeski, Second-order scalar-tensor field equations in a four-dimensional space, Int. J. Theor. Phys. 10, 363 (1974).

[34] A. Nicolis, R. Rattazzi, and E. Trincherini, Galileon as a local modification of gravity, Phys. Rev. D 79, 064036 (2009).

[35] C. Deffayet, G. Esposito-Farèse, and A. Vikman, Covariant Galileon, Phys. Rev. D 79, 084003 (2009).

[36] C. Deffayet, S. Deser, and G. Esposito-Farèse, Generalized Galileons: All scalar models whose curved background extensions maintain second-order field equations and stress tensors, Phys. Rev. D 80, 064015 (2009).

[37] T. Kobayashi, M. Yamaguchi, and J. Yokoyama, Generalized G-Inflation:- Inflation with the Most General SecondOrder Field Equations-, Prog. Theor. Phys. 126, 511 (2011).

[38] C. Deffayet and D. A. Steer, A formal introduction to Horndeski and Galileon theories and their generalizations, Classical Quant. Grav. 30, 214006 (2013).

[39] G. Dvali, G. Gabadadze, and M. Porrati, 4D gravity on a brane in 5D Minkowski space, Phys. Lett. B 485, 208 (2000).

[40] A. Nicolis and R. Rattazzi, Classical and quantum consistency of the DGP model, J. High Energy Phys. 06 (2004) 059.

[41] C. de Rham, Galileons in the skyGaliléons dans les cieux, C.R. Phys. 13, 666 (2012).

[42] C. de Rham, Massive gravity, Living Rev. Relativity 17, 7 (2014).

[43] K. Van Acoleyen and J. Van Doorsselaere, Galileons from Lovelock actions, Phys. Rev. D 83, 084025 (2011).

[44] C. Charmousis, From Lovelock to Horndeski's generalised scalar-tensor theory, in Proceedings of the 7th Aegean Summer School: Beyond Einstein's theory of gravity. Modifications of Einstein's Theory of Gravity at Large Distances, Paros, Greece, 2013, arXiv:1405.1612.

[45] D. Bettoni, J. M. Ezquiaga, K. Hinterbichler, and M. Zumalacárregui, Speed of gravitational waves and the fate of scalar-tensor gravity, Phys. Rev. D 95, 084029 (2017).

[46] P. Creminelli and F. Vernizzi, Dark Energy after GW170817 and GRB170817A, Phys. Rev. Lett. 119, 251302 (2017).

[47] J. M. Ezquiaga and M. Zumalacárregui, Dark Energy After GW170817: Dead Ends and the Road Ahead, Phys. Rev. Lett. 119, 251304 (2017).

[48] E. J. Copeland, M. Kopp, A. Padilla, P. M. Saffin, and C. Skordis, Dark Energy after GW170817 Revisited, Phys. Rev. Lett. 122, 061301 (2019).

[49] P. Brax, C. Burrage, and A.-C. Davis, Laboratory tests of the Galileon, J. Cosmol. Astropart. Phys. 09 (2011) 020.

[50] T. Kobayashi, H. Tashiro, and D. Suzuki, Evolution of linear cosmological perturbations and its observational implications in Galileon-type modified gravity, Phys. Rev. D 81, 063513 (2010).

[51] A. De Felice and S. Tsujikawa, Conditions for the cosmological viability of the most general scalar-tensor theories and their applications to extended Galileon dark energy models, J. Cosmol. Astropart. Phys. 02 (2012) 007.

[52] A. Barreira, B. Li, W. A. Hellwing, C. M. Baugh, and S. Pascoli, Nonlinear structure formation in the cubic Galileon gravity model, J. Cosmol. Astropart. Phys. 10 (2013) 027.

[53] E. Babichev and G. Esposito-Farèse, Time-dependent spherically symmetric covariant Galileons, Phys. Rev. D 87, 044032 (2013).

[54] E. Babichev and G. Esposito-Farèse, Cosmological selftuning and local solutions in generalized Horndeski theories, Phys. Rev. D 95, 024020 (2017).

[55] L. Hui and A. Nicolis, No-Hair Theorem for the Galileon, Phys. Rev. Lett. 110, 241104 (2013).

[56] T. P. Sotiriou and S. Zhou, Black Hole Hair in Generalized Scalar-Tensor Gravity, Phys. Rev. Lett. 112, 251102 (2014).

[57] T. P. Sotiriou and S. Zhou, Black hole hair in generalized scalar-tensor gravity: An explicit example, Phys. Rev. D 90, 124063 (2014).

[58] E. Babichev, C. Charmousis, and A. Lehébel, Black holes and stars in Horndeski theory, Classical Quant. Grav. 33, 154002 (2016).

[59] A. Maselli, H. O. Silva, M. Minamitsuji, and E. Berti, Slowly rotating black hole solutions in Horndeski gravity, Phys. Rev. D 92, 104049 (2015).

[60] A. Lehébel, E. Babichev, and C. Charmousis, A no-hair theorem for stars in Horndeski theories, J. Cosmol. Astropart. Phys. 07 (2017) 037.

[61] E. Babichev and C. Charmousis, Dressing a black hole with a time-dependent Galileon, J. High Energy Phys. 08 (2014) 106.

[62] E. Babichev, Galileon accretion, Phys. Rev. D 83, 024008 (2011).

[63] E. Babichev, C. Deffayet, and G. Esposito-Farèse, Constraints on Shift-Symmetric Scalar-Tensor Theories with a Vainshtein Mechanism from Bounds on the Time Variation of G, Phys. Rev. Lett. 107, 251102 (2011).

[64] K. Van Aelst, E. Gourgoulhon, P. Grandclément, and C. Charmousis, Hairy rotating black holes in cubic Galileon theory, Classical Quant. Grav. 37, 035007 (2020).

[65] E. Babichev, C. Charmousis, A. Lehébel, and T. Moskalets, Black holes in a cubic Galileon universe, J. Cosmol. Astropart. Phys. 09 (2016) 011.

[66] P. Boonserm, T. Ngampitipan, A. Simpson, and M. Visser, Innermost and outermost stable circular orbits in the presence of a positive cosmological constant, Phys. Rev. D 101, 024050 (2020).

[67] J. Khoury and A. Weltman, Chameleon Fields: Awaiting Surprises for Tests of Gravity in Space, Phys. Rev. Lett. 93, 171104 (2004).

[68] D. F. Mota and D. J. Shaw, Evading equivalence principle violations, cosmological, and other experimental constraints in scalar field theories with a strong coupling to matter, Phys. Rev. D 75, 063501 (2007).

[69] K. A. Olive and M. Pospelov, Environmental dependence of masses and coupling constants, Phys. Rev. D 77, 043524 (2008). 
[70] K. Hinterbichler and J. Khoury, Screening Long-Range Forces through Local Symmetry Restoration, Phys. Rev. Lett. 104, 231301 (2010).

[71] E. Babichev, C. Deffayet, and R. Ziour, k-mouflage gravity, Int. J. Mod. Phys. D 18, 2147 (2009).

[72] K. Hinterbichler, Theoretical aspects of massive gravity, Rev. Mod. Phys. 84, 671 (2012).

[73] E. Babichev and C. Deffayet, An introduction to the Vainshtein mechanism, Classical Quant. Grav. 30, 184001 (2013).

[74] F. H. Vincent, T. Paumard, E. Gourgoulhon, and G. Perrin, Classical and Quantum Gravity PAPERS GYOTO: A new general relativistic ray-tracing code, Classical Quant. Grav. 28, 225011 (2011).

[75] F. H. Vincent, E. Gourgoulhon, and J. Novak, $3+1$ geodesic equation and images in numerical spacetimes, Classical Quant. Grav. 29, 245005 (2012).

[76] K. Glampedakis and G. Pappas, Can supermassive black hole shadows test the Kerr metric?, Phys. Rev. D 104, L081503 (2021).

[77] O. Straub, F. H. Vincent, M. A. Abramowicz, E. Gourgoulhon, and T. Paumard, Modelling the black hole silhouette in Sagittarius A* with ion tori, Astron. Astrophys. 543, A83 (2012).

[78] M. A. Abramowicz and P. C. Fragile, Foundations of black hole accretion disk theory, Living Rev. Relativity 16, 1 (2013).

[79] F. H. Vincent, W. Yan, O. Straub, A. A. Zdziarski, and M. A. Abramowicz, A magnetized torus for modeling Sagittarius A* millimeter images and spectra, Astron. Astrophys. 574, A48 (2015).

[80] F. Yuan and R. Narayan, Hot accretion flows around black holes, Annu. Rev. Astron. Astrophys. 52, 529 (2014).

[81] A. Pandya, Z. Zhang, M. Chandra, and C. F. Gammie, Polarized synchrotron emissivities and absorptivities for relativistic thermal, power-law, and kappa distribution functions, Astrophys. J. 822, 34 (2016).

[82] H.-Y. Pu and A.E. Broderick, Probing the innermost accretion flow geometry of Sgr A* with Event Horizon Telescope, Astrophys. J. 863, 148 (2018).

[83] G. C. Bower et al., ALMA polarimetry of Sgr A*: Probing the accretion flow from the event horizon to the bondi radius, Astrophys. J. 868, 101 (2018).

[84] Y. Kato, M. Miyoshi, R. Takahashi, H. Negoro, and R. Matsumoto, Measuring spin of a supermassive black hole at the Galactic centre-implications for a unique spin, Mon. Not. R. Astron. Soc. 403, L74 (2010).

[85] R. Nemmen, The Spin of M87*, Astrophys. J. 880, L26 (2019).

[86] F. Tamburini, B. Thidé, and M. Della Valle, Measurement of the spin of the M87 black hole from its observed twisted light, Mon. Not. R. Astron. Soc. 492, L22 (2020).

[87] G. Fragione and A. Loeb, An upper limit on the spin of SgrA* based on stellar orbits in its vicinity, Astrophys. J. 901, L32 (2020).

[88] J. Dexter et al. (GRAVITY Collaboration), The resolved size and structure of hot dust in the immediate vicinity of AGN, Astron. Astrophys. 635, A92 (2020).

[89] L. Barack and C. Cutler, LISA capture sources: Approximate waveforms, signal-to-noise ratios, and parameter estimation accuracy, Phys. Rev. D 69, 082005 (2004).

[90] S. Babak, J. Gair, A. Sesana, E. Barausse, C. F. Sopuerta, C. P. L. Berry, E. Berti, P. Amaro-Seoane, A. Petiteau, and A. Klein, Science with the space-based interferometer LISA. V. Extreme mass-ratio inspirals, Phys. Rev. D 95, 103012 (2017).

[91] S. E. Gralla, A. Lupsasca, and D. P. Marrone, The shape of the black hole photon ring: A precise test of strong-field general relativity, Phys. Rev. D 102, 124004 (2020).

[92] C. Bambi, Astrophysical black holes: A review, Proc. Sci., MULTIF2019 (2020) 028.

[93] Z. Zhu, M. D. Johnson, and R. Narayan, Testing general relativity with the black hole shadow size and asymmetry of sagittarius $A^{*}$ : Limitations from interstellar scattering, Astrophys. J. 870, 6 (2018).

[94] S. Issaoun et al., Persistent non-gaussian structure in the image of sagittarius $\mathrm{A}^{*}$ at $86 \mathrm{GHz}$, Astrophys. J. 915, 99 (2021).

[95] J. Bardeen, Stability of circular orbits in stationary, axisymmetric space-times, Astrophys. J. 161, 103 (1970).

[96] J. Bardeen, W. Press, and S. Teukolsky, Rotating black holes: Locally nonrotating frames, energy extraction, and scalar synchrotron radiation, Astrophys. J. 178, 347 (1972).

[97] K. Van Aelst, Note on equatorial geodesics in circular spacetimes, Classical Quant. Grav. 37, 207001 (2020).

[98] E. Gourgoulhon, An introduction to the theory of rotating relativistic stars, in Proceedings of CompStar 2010: School and Workshop on Computational Tools for Compact Star Astrophysics Ganil, Caen, France, 2010, arXiv:1003.5015. 